\title{
Macrolides for yet another chronic airway disease: severe asthma?
}

Peter G Gibson

Macrolides have a macrocyclic lactone ring, whose size and features have been modified from the 14 carbon structure of erythromycin in order to develop newer agents such as azithromycin (AZM). They were originally derived from products the microbial order Actinomycetales (Saccharopolyspora erythrae, formerly Streptomyces erythrae), and are yet another respiratory harvest from the organism that have given us many antibiotics, starting with the important antituberculosis drug, streptomycin. Macrolides are widely used as antibiotics, and now form part of guideline-recommended therapy in community-acquired pneumonia. They have an expanding role in the therapy of chronic inflammatory diseases based on their additional anti-inflammatory and immunosuppressive properties. Further development has produced broad-spectrum antibacterials, such as AZM, with a longer half-life, and minimal inhibition of cytochrome CYP3A4 and, hence, fewer drug interactions. Other developments in this class include tacrolimus and its derivatives that target macrophyllin-12 (FK506 binding protein) and are potent immunosuppressive agents.

The classical airway disease that responds to long-term low-dose macrolide therapy is diffuse panbronchiolitis in Asian populations. ${ }^{1}$ The pathological lesion is centred around the bronchioles and involves intense chronic inflammation, typically neutrophilic, leading to mucus hypersecretion, progressive loss of lung function, bacterial airway infection, and often death. Building on this paradigm, macrolides have been found to be effective in other airway diseases, such as post-transplant bronchiolitis obliterans syndrome, bronchiectasis, cystic fibrosis and, recently, chronic obstructive pulmonary disease (COPD). ${ }^{2}$ Neutrophilic bronchitis and bronchiolitis are common features of these diseases and may represent the target lesion. The most consistent effect of macrolides in these disorders is a reduction in exacerbation rate, with variable effects on lung function, symptoms and mucus hypersecretion. The recognition that some forms of asthma, particularly severe

Correspondence to Dr Peter G Gibson, Department of Respiratory and Sleep Medicine, John Hunter Hospital, Newcastle, NSW 2310, Australia; peter.gibson@newcastle.edu.au asthma, may have a neutrophilic bronchitis ${ }^{3}$ has prompted a re-evaluation of macrolides in asthma.

Earlier studies that evaluated macrolides in the non-eosinophilic severe asthma phenotype were positive for their respective outcomes, ${ }^{4} 5$ whereas studies in milder forms of asthma, or non-phenotyped populations, yield negative or mixed results. ${ }^{6}$ Against this background, the AZIAST study provides further evidence to support the efficacy of AZM in severe non-eosinophilic asthma, but there are qualifications, requiring further studies. ${ }^{7}$

The randomised trial was methodologically well conducted, with good retention, and used clinically relevant outcome measures. The subjects had symptomatic asthma despite taking a high-dose inhaled corticosteroid and long-acting B2 agonist, with a long history of disease, and were selected to have at least two severe asthma exacerbations and/or lower respiratory tract infections (LRTI) in the prior year. They are the type of patient where add-on therapy should be considered, but so should optimisation of self-management skills, including adherence, and assessment and treatment of comorbidity. Self-management skills and inhaled corticosteroid (ICS) adherence were optimised, and the low fractional concentration of exhaled nitric oxide (FENO) at entry is consistent with a low rate of ICS non-adherence. Comorbidities were not specifically addressed prior to entry, and perhaps further research should be carried out to investigate the relative benefit of management based on clinical phenotype (including assessment and treatment of comorbidities) versus management based on inflammatory phenotype.

There was no effect of AZM on the primary outcome in the total population when assessed without inflammatory phenotyping. This is an important negative result for several reasons. First, the subject selection included a requirement for prior LRTIs, and it could be argued that an antibiotic will of course reduce episodes of infective bronchitis. The fact that this did not occur in the group overall (but did occur in the non-eosinophilic subphenotype) supports a phenotype-specific effect, occurring by mechanisms other than the antibacterial activity of AZM. Second, the result underscores the need to phenotype patients in trials of severe asthma when the aim is to assess the effects of pathwayspecific therapies. The risk of not doing this is to falsely dismiss a treatment. The result also shows the need to phenotype patients in order to prevent unnecessary exposure of the patient to a drug that may be ineffective or even harmful for them.

The drug had differential effects in the different asthma inflammatory phenotypes. AZM reduced severe asthma exacerbations and the compound endpoint of exacerbation and/or LRTI in the subphenotype of non-eosinophilic asthma. The effect was highly clinically significant, with approximately $67 \%$ of AZM-treated patients remaining exacerbation and/or infection free, compared with $38 \%$ in the placebo group. The non-eosinophilic group was relatively common, and importantly, was not recognised by FENO alone. At least half the study population had eosinophilic asthma with low FENO. This discordance between airway-derived FENO levels and blood eosinophilia in severe asthma has been noted previously, and probably relates to the specificity of FENO for steroid-sensitive epithelial i nitric oxide synthase (iNOS) changes, and not the bone marrow lesion that is a recognisable component of refractory eosinophilic asthma.

AZM was ineffective in eosinophilic asthma, and in fact, the eosinophilic subgroup had more exacerbations when taking AZM. This is both unexpected and unexplained, but warrants follow-up. The drug was well tolerated, but concerns remain with the effect of this therapy on community-wide rates of macrolide resistance. These issues will need to be addressed through more research. Potential cardiac toxicity was avoided by subject screening prior to study entry and monitoring during the study. These are essential components of managing a patient on long-term macrolide therapy.

The purported mechanisms of AZM in non-eosinophilic severe asthma include antibacterial activity against an underlying chronic infection, such as Chlamydia pneumoniae, antineutrophil activity either by enhanced clearance of neutrophils (macrophage efferocytosis) ${ }^{8}$ or increased neutrophil apoptosis, or an effect on airway mucus. The study found no effect based on the presence or absence of Cpn seropositivity (supplemental figure S2 in Brusselle et al). ${ }^{7}$ Recently noneosinophilic asthma has been shown to have impaired efferocytosis, ${ }^{9}$ and in COPD this is improved by $\mathrm{AZM},{ }^{8}$ supporting this mechanism of action in AZIAST.

The AZISAST study strongly supports the need to use inflammatory 
phenotyping when evaluating new therapies in severe asthma. It provides promising data for a clinically important but phenotype-specific effect of AZM in non-eosinophilic asthma, but also highlights a potential downside of microbial resistance. Before recommending AZM for severe asthma, we need more of both efficacy and safety data from larger patient numbers, and from a primary outcome analysis. Such a study is ongoing (ACTRN12609000197235).

\section{Competing interests None.}

Provenance and peer review Commissioned; internally peer reviewed.

To cite Gibson PG. Thorax 2013;68:313-314.

Received 17 December 2012

Accepted 17 December 2012

Published Online First 24 January 2013

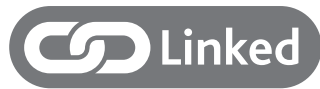

- http://dx.doi.org/10.1136/thoraxjnl-2012-202698

Thorax 2013;68:313-314.

doi:10.1136/thoraxjnl-2012-203055

\section{REFERENCES}

1 Koyama $\mathrm{H}$, Geddes DM. Erythromycin and diffuse panbronchiolitis. Thorax 1997;52:915-18.

2 Crosbie PAJ, Woodhead M. Long-term macrolide therapy in chronic inflammatory airway diseases. Eur Resp J 2004;24:822-33.

3 Green RH, Brightling CE, Woltmann G, et al. Analysis of induced sputum in adults with asthma: identification of a subgroup with isolated sputum neutrophilia and poor response to inhaled corticosteroids. Thorax 2002;57:875-9.

4 Coeman M, van Durme Y, Bauters F, et al. Neomacrolides in the treatment of patients with severe asthma and/or bronchiectasis: a retrospective observational study. Ther Advances in Resp Dis 2011;5:377-86.

5 Simpson JL, Powell H, Boyle MJ, et al. Clarithromycin targets neutrophilic airway inflammation in refractory asthma. Am J Resp Crit Care Med 2008;177:148-55.

6 Sutherland ER, King TS, Icitovic N, et al. A trial of clarithromycin for the treatment of suboptimally controlled asthma. J Allergy Clin Immunol 2010;126:747-53.

7 Brusselle G, Jordens P, Deman R, et al. Azithromycin for prevention of exacerbations in severe asthma (AZISAST): a multicentre, randomized, double-blind, placebo-controlled trial. Thorax Published Online First: 3 January 2013. doi:10.1136/thoraxjnl-2012-202698

8 Hodge $\mathrm{S}$, Hodge $\mathrm{G}$, Jersmann $\mathrm{H}$, et al. Azithromycin improves macrophage phagocytic function and expression of mannose receptor in chronic obstructive pulmonary disease. Am J Respir Crit Care Med 2008;17:139-48.

9 Simpson JL, Gibson PG, Yang IA, et al. AMAZES Study Research Group. Impaired macrophage phagocytosis in non-eosinophilic asthma. Clin Exp Allergy 2013;43:29-35. 